\title{
Continuous mapping of the cortical object vision pathway using traveling waves in object space
}

\author{
Elfi Goesaert ${ }^{\mathrm{a}}$, Hans P. Op de Beeck ${ }^{\mathrm{a}, \mathrm{b}, *}$ \\ a Laboratory of Experimental Psychology, University of Leuven (K.U.Leuven), Tiensestraat 102, 3000 Leuven, Belgium \\ ${ }^{\mathrm{b}}$ Laboratory of Biological Psychology, University of Leuven (K.U.Leuven), Tiensestraat 102, 3000 Leuven, Belgium
}

\section{A R T I C L E I N F O}

\section{Article history:}

Received 3 September 2009

Revised 30 October 2009

Accepted 16 November 2009

Available online $\mathrm{xxxx}$

\begin{abstract}
A B S T R A C T
Research into the organization of high-level visual cortex has mainly used discrete object classes (e.g. faces and buildings). In contrast, the lower levels of the visual cortex have mainly been studied using phaseencoding techniques with gradually changing rotating wedges and expanding/contracting rings. The gradual stimulus changes cause traveling waves on the cortical surface and time delays of each cortical location are related to stimulus preference. We propose to extend this method to study higher-order properties of visual objects. We created gradual morphing sequences from a face into a building and vice versa. The activation in traditional face- and house-selective regions corresponding to fusiform face area (FFA) and parahippocampal place area (PPA) was time-locked to presentation of respectively face(-like) and building(-like) stimuli in the morphing sequences. We also included contracting/expanding rings containing images of parts of natural scenes. Confirming existing literature with discrete object classes, activity in FFA and PPA was time-locked to the presentation of foveal and eccentric rings, respectively. The results however contradict large-scale continuous maps across the ventral visual stream, as the cortical area between FFA and PPA was not modulated by the position in the morphing sequences, or by the retinotopic position of the rings. We conclude that phase-encoding techniques can help in revealing the organization of high-level visual regions for complex object properties.
\end{abstract}

(c) 2009 Elsevier Inc. All rights reserved.

\section{Introduction}

The higher visual regions in the human occipitotemporal cortex are involved in the recognition of objects and faces. These regions contain a variety of response profiles. A large cortical region responds more strongly to images of intact objects than to scrambled images (lateral occipital complex or LOC; see Malach et al., 1995; Grill-Spector et al., 1998; Murray et al., 2002; Grill-Spector and Malach, 2004). More focal regions respond preferentially to exemplars from specific object categories, such as faces (including the occipital and fusiform face areas or OFA/FFA; see Kanwisher et al., 1997; Grill-Spector, 2003; Kanwisher, 2006), body parts (including the extrastriate body area or EBA; see Downing et al., 2001; Peelen and Downing, 2007; Schwarzlose et al., 2005), or places/houses/landscapes (particularly in the parahippocampal place area or PPA, see Epstein and Kanwisher 1998; Epstein et al., 1999).

The question arises whether these seemingly category-selective regions operate as functionally separate units (modular organization), or whether there is an underlying organization connecting all these regions together (Op de Beeck et al., 2008a). One possibility is that

\footnotetext{
* Corresponding author. Laboratory of Biological Psychology, University of Leuven (K U.Leuven), Tiensestraat 102, B3000 Leuven, Belgium.

E-mail address: hans.opdebeeck@psy.kuleuven.be (H.P. Op de Beeck)
}

these apparently distinct regions would in fact be the most easily detected parts of a larger and more continuous topographic map. In such a map we expect the activation to shift across the ventral part of the visual cortex as the properties of the objects change. This is a fundamental property of topographic maps, which in the visual system is most clearly illustrated by the retinotopic maps in primary visual cortex and surrounding areas (DeYoe et al., 1994; Engel et al., 1994; Schira et al., 2009; Sereno, 1998; Sereno et al., 1994; Tootell et al., 1997; Wandell, 1999; Wandell and Wade, 2003).

In the present study we propose a methodology to study the presence of a topographic map in high-level visual cortex that connects regions with a preference for different object categories. Continuous maps can only be uncovered by a continuous change in the stimuli. For example, the large-scale retinotopic organization (for eccentricity and polar angle) of the early visual cortex has been studied using phase-encoding techniques, where the preference for continuously changing stimuli is mapped onto the cortex, creating traveling waves of activation across the cortical surface. In these studies, the stimuli are created to optimally activate the lower visual regions, using rotating wedges and expanding or contracting rings filled with a highcontrast checkerboard pattern. There have been few studies that adapted this paradigm to fit higher-level areas of the cortex. For example, a study by Saygin and Sereno (2008) used complex visual stimuli to fill up the wedges and rings and manipulated attention to 
the stimuli to activate the parietal cortex. Nevertheless, the property of interest was still stimulus position on the visual field and the retinotopic organization of the activated cortex. Puce et al. (1996) used Fourier analysis to investigate categorizing processes in the ventral visual cortex, however, the paradigm used was different from phase-encoded mapping. We would like to investigate the functional organization of the higher visual cortex for other stimulus features, such as object categories, using this specific technique.

To successfully investigate the functional organization for these stimulus features, it might be useful to apply the phase-encoding techniques to these features as well. This new application implies the construction of specific stimuli that show a gradual shift between these features, for example, a shift between object categories. The object of this study was to do just that: We are taking specific object categories (faces and houses), and gradually changing them from one category to another, to investigate whether this gradual shift in the stimuli would result in a gradual activation shift across the cortex. The choice fell on houses and faces because there is compelling evidence that regions like the FFA and the PPA respond stronger to faces and houses, respectively, than to other objects. By choosing object categories that cause activations on different but not too remote locations of the cortex, it should be easier to spot possible shifts in activation across the cortex. Tootell et al. (2008) have, in a previous study, used morph stimuli of faces and houses and applied single stimulus imaging to look into the matter of intermediate activation. The study differed greatly from our approach in certain respects: Morph stimuli used consisted of unidentifiable objects, and the method used was again a standard block design. The main focus of our study was to use phase-encoding techniques, first, to establish a new method useful in studying highlevel visual cortex and its organization and second, to be able to more directly compare the results with traditional mapping studies.

During this investigation we cannot ignore another unknown about the functional organization of the cortical regions showing category-selective responses. Whereas it is tempting to refer to object category as the fundamental functional property that underlies the selectivity, several other and simpler properties have been proposed that might contribute to the selectivity related to category membership. These properties include perceived shape (Haxby et al., 2000; Op de Beeck et al., 2008b), cognitive processes (Gauthier et al., 2000), and semantic attributes (Martin and Chao, 2001), but the simplest is the proposal of a weak retinotopic organization (Levy et al., 2001; Haason et al., 2002 and Malach et al., 2002 for an overview). Some of these object properties might be organized in terms of a topographic map, and others not. One possibility, suggested before (Op de Beeck et al., 2008a) is that the category selectivity itself might be focal and discontinuous while the simpler properties might be organized in smoother, possibly topographically organized maps. Fig. 1 shows a schematic representation of modularity vs topographic maps. Where modular organization shows a sudden change in amplitude, the strength of selectivity is much more gradual when looking at topographic maps. So we investigated the presence of topographic maps not only for complex object properties but also for eccentricity, arguably the simplest of these alternative properties.

Summarized, the objectives of the present study are to (i) apply phase-encoding techniques to the functional selectivity for object categories; (ii) validate the results by comparing them with selectivity as seen in a traditional block design; (iii) investigate to what extent a continuous topographic map exists for this complex object property; and (iv) compare the cortical mapping of object categories with the mapping of eccentricity.

\section{Materials and methods}

\section{Subjects}

Two subjects participated in the fMRI study (one male aged 28 years, one female aged 22 years). They had previous experience as subjects in fMRI studies, signed an informed consent form and were compensated for their participation. The study was approved by the relevant ethical boards (ethical committee of the Faculty of Psychology and Educational Sciences and the committee for medical ethics of the Katholieke Universiteit Leuven, Leuven, Belgium).
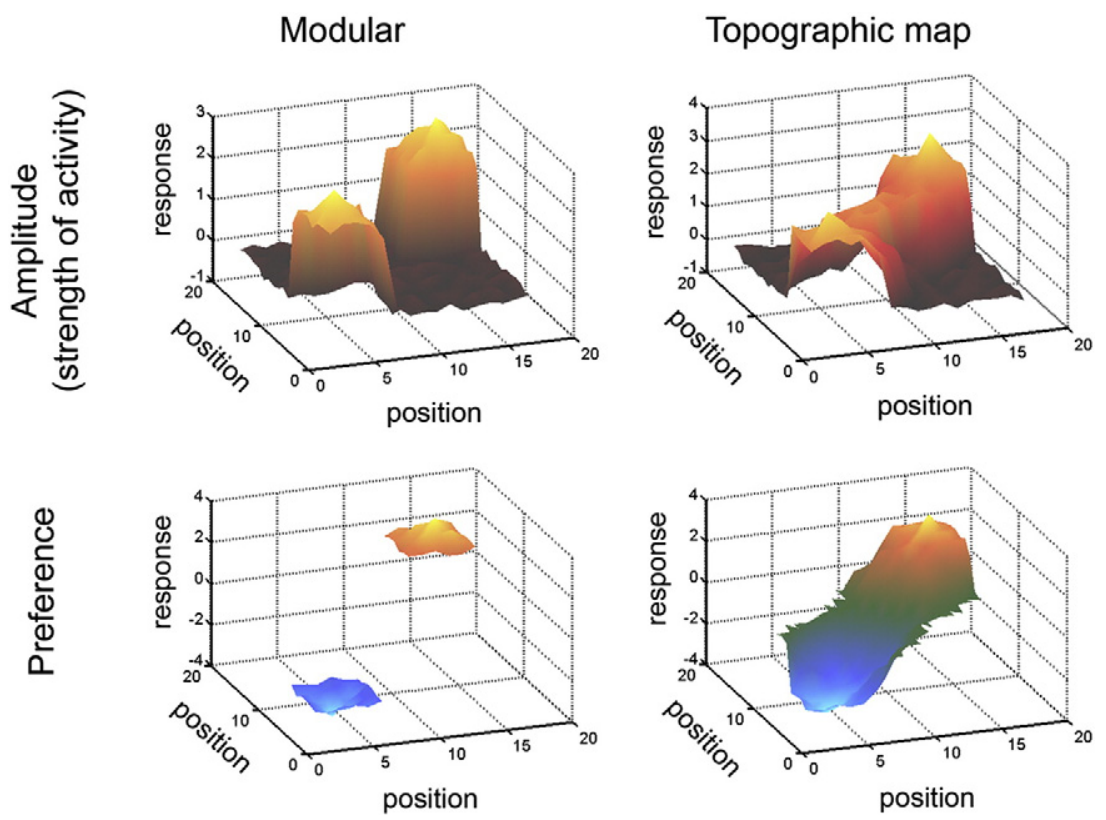

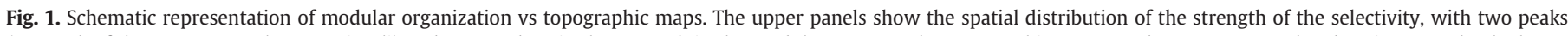

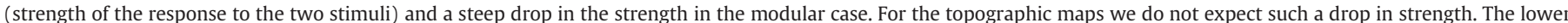

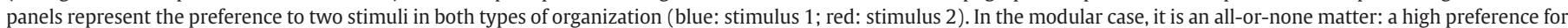

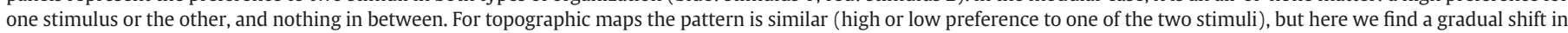
preference when moving from one stimulus to another. 
We also performed a behavioral pilot study, in which 10 subjects participated ( 5 females, aged 19-45 years). They either volunteered or were granted course credit for their participation, and half of them were naive subjects ( 2 subjects were the authors, and 3 subjects were unfamiliar with the present research but were experienced in perception research in general).

\section{Stimuli}

There were two parts to the study: the object morph stimuli using a face and a house (Fig. 2a), and the retinotopic stimuli (Fig. 2b) consisting of rings that were shown as expanding or contracting.

The morph stimuli were created in 3DS Max (Autodesk, San Rafael, CA). A default face was created, and used as a template for the house stimulus. Both objects were subsequently linked, morphed, and matched for surface reflectance. We also matched the stimuli as closely as possible in retinotopic extent. We created a series of 100 images ranging from $100 \%$ face to $100 \%$ house. The morphing algorithm implemented in 3DS Max does not guarantee that the subsequent steps represent the same physical change. We therefore selected 24 stimuli based on a pixel-based measure of similarity between subsequent pairs: We computed the total squared difference of the images, and the images selected showed approximately the same level of difference from one image to the next step in the 24 image sequence. The images were grayscaled and were 2.5 by $3.5 \mathrm{~cm}$ (corresponding to a retinal stimulus size between 4 and 6 visual degrees). There were three possible orientations to the stimuli $\left(45^{\circ}\right.$ left, $45^{\circ}$ right and front view). Fig. 3 shows examples of these three possible orientations for three steps in the morphing process: $100 \%$ face, $100 \%$ house and $50 \%$ face $/ 50 \%$ house.

The retinotopic stimuli also consisted of 24 images, this time rings with a thickness of about $1 \mathrm{~cm}$, varying in size from about $1.5 \mathrm{~cm}$ in diameter to $11 \mathrm{~cm}$ in diameter, corresponding to a retinal stimulus size between 2.5 and 18 visual degrees. Inside these rings scenes of objects were presented (repetitions of faces, buildings, penguins, butterflies, yoghurt containers, wine bottles, sheep and books). The order of presentation of these objects was randomized. There was no repetition of an object in subsequent rings, and each set of objects appeared an equal amount (18 times over 6 cycles). The experiments were programmed using MATLAB (Mathworks, Natick, MA) and PsychToolbox (Brainard, 1997) was used for the presentation of the stimuli during the experiment, as well as the response registration. We used a Barco 6400i LCD projector (resolution $1027 \times 768$, refresh rate $75 \mathrm{~Hz}$ ) to project the stimuli on a screen, which was positioned approximately $35 \mathrm{~cm}$ from the subjects' eyes. The projection on the screen was made visible to the subjects during scanning by means of a mirror in front of their head.
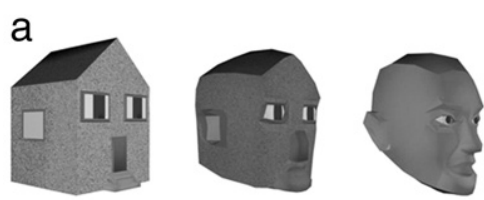

b
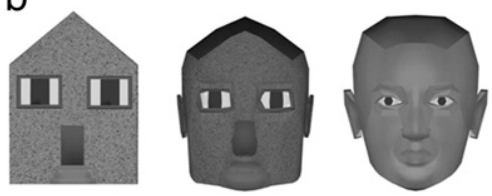

C
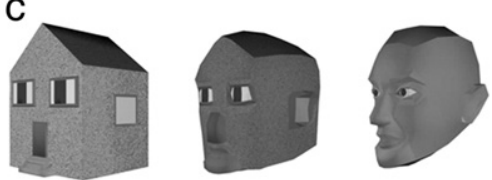

Fig. 3. The three different orientations of the morph series for $100 \%$ house, $100 \%$ face and the 50/50 morph, at either (a) $45^{\circ}$ left angle, (b) front view, or (c) $45^{\circ}$ right angle.

\section{Experiments}

\section{fMRI: Scanning}

Functional imaging data were collected using a 3T Philips Intera magnet (Department of Radiology, Katholieke Universiteit Leuven, Leuven, Belgium) with an 8-channel SENSE head coil with an echoplanar imaging sequence (86 time points per time series or "run"; repetition time, $2000 \mathrm{~ms}$; echo time, $29.8 \mathrm{~ms}$; acquisition matrix $104 \times 104$, resulting in a 2.0 by $2.0 \mathrm{~mm}^{2}$ in-plane voxel size; 33 slices oriented approximately halfway between a coronal and horizontal orientation and including most of cortex except the most superior parts of frontal and parietal cortex, with slice thickness $2 \mathrm{~mm}$ and interslice gap $0.2 \mathrm{~mm}$ ). We also acquired a T1-weighted anatomical image (resolution 0.98 by 0.98 by $1.2 \mathrm{~mm}, 9.6 \mathrm{~ms}$ TR, $4.6 \mathrm{~ms} \mathrm{TE}$, $256 \times 256$ acquisition matrix, 182 coronal slices).

\section{fMRI: Experimental runs}

During a 2 h scan session, 16 runs were recorded. One run started with a period of fixation ( $14 \mathrm{~s}$ ), followed by 6 cycles, and again ending with a fixation period $(14 \mathrm{~s})$. One cycle consisted of a series of 24 stimuli, each presented $500 \mathrm{~ms}$, followed by a fixation period of $500 \mathrm{~ms}$, resulting in a cycle with a duration of $24 \mathrm{~s}$. This resulted in a frequency of $1 / 12 \mathrm{~Hz}$ for the presentation of the stimuli, and the duration of a total run was $172 \mathrm{~s}$.

In the object morph condition, one cycle of 24 stimuli showed a series of gradual morphs from a face to a house, or from a house to a

a
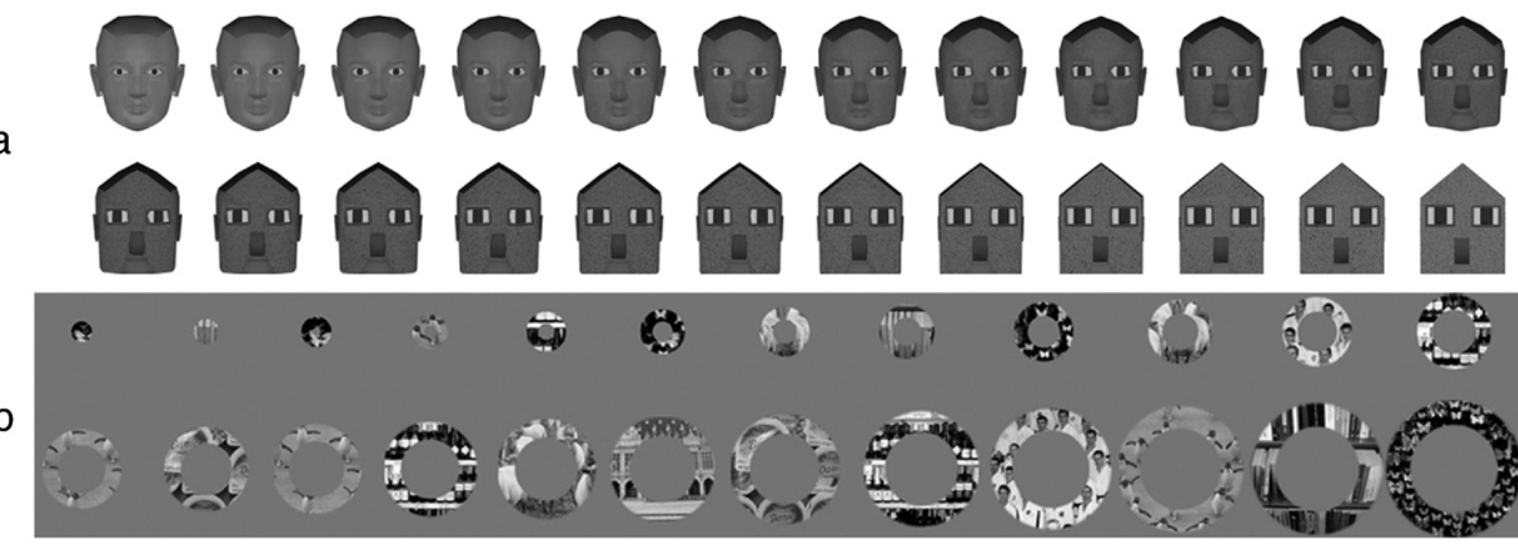

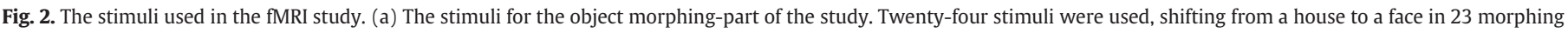

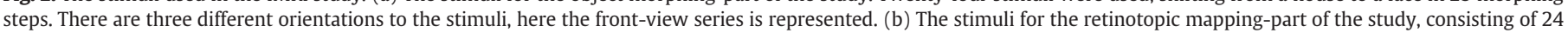
rings of gradually changing size, filled with scenes containing objects such as butterflies, buildings, people, sheep, wine bottles, etc. 
face. Each run ( 6 cycles) consisted of only one kind of orientation $\left(45^{\circ}\right.$ left, $45^{\circ}$ right or front view) in one particular order (face to house or house to face). The stimuli varied in location around a fixation point (maximal position offset range between 8 and 9 visual degrees), to eliminate a possible eccentricity bias and counter possible slight differences in retinotopic extent between the face and house stimuli. When a face had completely morphed into a house, the next stimulus was again a face stimulus starting to morph back to a house shape.

In the contracting/expanding rings condition, a series of 24 increasingly larger or smaller rings moved systematically across the visual field to create a traveling wave in visual space. At the end of a cycle, a new one started. For example, if a contracting ring reached the center, the next stimulus appeared at the edges of the display, and started moving inwards again.

There were always two types of morph runs, and two types of eccentricity runs. The two types were opposites of each other, with an expanding-ring run followed by a contracting-ring run, and a facehouse run followed by a house-face run (or the other way around). The morph runs and retinotopic runs were alternated, and the order of the runs was counter-balanced across subjects, while also randomizing the viewpoint (left, right, and front) in the case of the morph stimuli.

Different colors (low saturated blue, red and green) were presented on the grayscaled images during the experiment. Subjects were asked to push a button when the color of the images changed (see also: Op de Beeck et al., 2006; Op de Beeck et al., 2008b, for use of the same task). The color changed about three times in the course of each run. This was to keep the subjects occupied during the experiment, and have them pay attention to the stimuli, without them having to attend to the specific content of the stimuli. One reason for not having them attend the specific content of the stimuli was to try and counter possible bistability when perceiving the morphs. Since the morphs contained clear features of both houses and faces, subjects might categorize all morphs, also the 50\% morphs, as either face or house. By focusing the attention away from the actual category or identity of the stimuli, we could attempt to investigate whether the bistability happened automatically on a neural level (in which case we would find a modular organization) or whether it was something that was linked to the conscious processing and categorization of the stimuli (in which case we might find a continuous organization in the cortex). The task was fairly easy, with subjects performing at a mean of $92 \%$ correct responses. Subjects were asked to fixate during the experimental runs. Eye movement data could only be acquired for one subject. An MR-compatible eye-tracking device (Applied Science Laboratories system 5000) reliably recorded the position of the left pupil during eight of the experimental runs. We performed a drift correction for each of these runs, and calculated a weighted mean and standard deviation across runs. We found that the position of the pupil did not deviate greatly from the fixation point, indicating good fixation during the course of the experiment $\left(\mathrm{SD}_{\text {horizontal movements }}=0.25, \mathrm{SD}_{\text {vertical movements }}=0.26\right.$, measured in visual degrees). Note that bad fixation would have worked against finding a clear retinotopic map in visual areas, but such a map was very prominent in each subject.

\section{fMRI: 3D Fourier analysis of the time series}

The periodically changing stimuli are expected to cause a dynamically changing pattern of neural activity, with a different timing of activation at locations that contain neurons with a different sensitivity (e.g. neurons with peripheral versus foveal receptive fields). Since this delay is periodical, it can be measured by the phase of a sinusoidal modulation of the fMRI signal (Engel et al., 1997). The phase was measured as follows. Each run was normalized to a mean of 0 , and a drift correction (linear and quadratic) was performed. Given the results for the pilot data, we averaged the runs for the three different orientations together. This resulted in one mean value per timepoint ( $T R=2000 \mathrm{~ms}$ ) per voxel for each direction of presentation (face to house or house to face). A Fourier analysis was then performed on each of these two time series per voxel, determining the phase and amplitude at the stimulus frequency ( 6 cycles per run of $72 \mathrm{~s}$, or $1 / 12 \mathrm{~Hz}$ ), taking into account the delay of the hemodynamic response (a shift of two TRs or $6 \mathrm{~s}$ was used as a first approximation). The phase values of the opposite directions of the stimuli (face to house and house to face; contracting and expanding) were averaged after reversing the sign and unwrapping the phase responses (Sereno et al., 1995; Warnking et al., 2002). This mean phase response was transformed to fit a scale from 0 to $2 *$ pi. This scale was color-coded and matched with the stimuli corresponding to that phase.

The data normalized to a mean of 0 were used for the Fourier analysis, but in order to find out the response strength to preferred and non-preferred images we also converted these values to percent signal change (PSC) values by adding the amplitude modulation to the difference in mean response during stimulus presentation and during the long fixation period at the beginning and end of the runs (baseline).

\section{fMRI: Regions of interest}

The FFA and PPA were defined using data from a previous experiment (Op de Beeck, Brants, Baeck and Wagemans, in press). Blocks of faces, houses and body parts were shown to the same two subjects who were asked to perform a one-back task during this experiment. The blocks consisted of 20 exemplars of a subordinate category (old/young faces, torsos/hands, old/modern buildings), and were presented for $15 \mathrm{~s}$. The analysis of the data was performed using SPM5 (Wellcome Department of Cognitive Neurology, London). The data were preprocessed, where the images were first corrected for differences in acquisition time, and then realigned to correct for small head movements. We co-registered the functional data with their anatomical image. The co-registered anatomical image was segmented, and from this step parameters were taken from which the functional images were spatially normalized to MNI space. The voxels were re-sampled to a voxel size of $2 \times 2 \times 2 \mathrm{~mm}$.

ROIs were defined by contrasting the face conditions versus houses and body parts conditions (FFA), and house conditions with faces and body parts conditions (PPA). These contrasts were calculated using SPM5 software and thresholded ( $p<0.0001$ uncorrected voxel level) and then mapped onto the cortex with CARET software (Van Essen Laboratory, Saint Louis, MO). We selected all significant voxels in a wide cortical area in ventral occipital and temporal cortex. Especially for the FFA this results in an ROI with multiple spots, some of which are outside the fusiform gyrus. Given our emphasis on the global aspects of object selectivity and eccentricity bias in ventral cortex, this broader definition of the ROI is more informative and preferable. The ROIs were defined prior to obtaining the data from the reported phase-encoding experiments.

\section{fMRI: Construction of the cortical model}

Topographic maps are clearest in an inflated or flattened view of the cortex (Warnking et al., 2002). An inflated view has the advantage that the cortex inside sulci is also visible. The anatomical image was segmented in CARET, separating white matter, gray matter and cerebrospinal fluid, for each hemisphere. The ROIs obtained in a previous experiment were co-registered with the anatomical scan and the newly obtained data and mapped onto the inflated cortex, creating a ventral face-selective region containing FFA and OFA, and a house-selective region containing PPA. Subsequently, the phase responses were displayed onto the inflated cortex. These phase responses were thresholded to contain only the strongest responses. To threshold these responses, we used the response amplitude corresponding to the stimulus frequency. It was calculated by dividing the amplitude of the Fourier component that matched the frequency of the stimulus pattern with the total Fourier power. This was done for both directions of the runs (each consisting of 4 time series), and we 


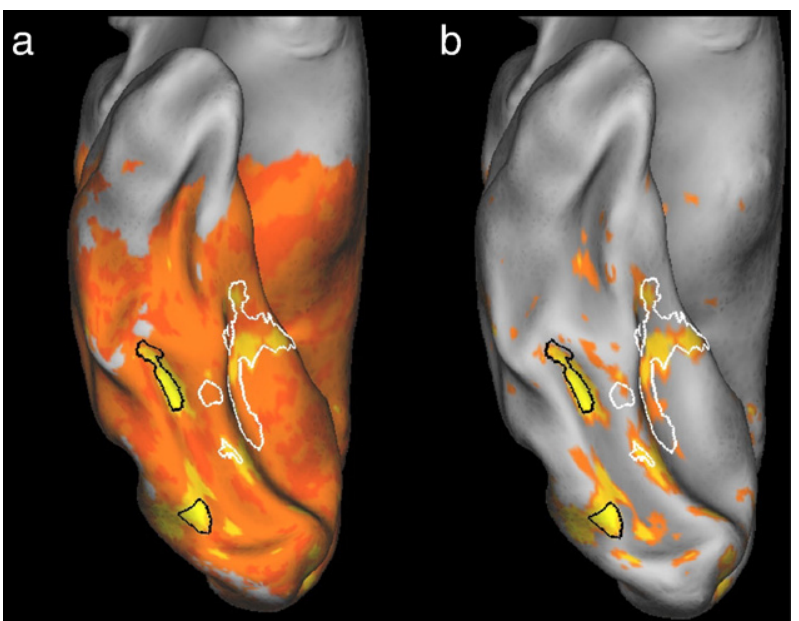

Fig. 4. Presentation of amplitudes for subject 1. (a) The inflated right hemisphere in a ventral view. FFA is shown in black, PPA in white. The strongest amplitudes to the morph stimuli are in yellow, and darken with diminishing strength. (b) The same inflated right hemisphere after the amplitudes is thresholded.

then calculated an average amplitude across voxels for the two conditions. This Fourier analysis and subsequent extraction of response amplitude was also performed on a selection of voxels of both white matter regions and areas located outside the brain to establish an amplitude threshold. We then compared the corrected measures of response magnitude for the ROIs with the histograms of response magnitudes in the selected white matter and outside-brain voxels. This resulted in a cutoff point for each subject and each condition (eccentricity or morph stimuli) corresponding to $p<0.05$ in the white matter ROI and the outside-the-brain ROI (uncorrected for multiple comparisons). When looking at the cutoff points established based on comparison with the white matter regions and the outsidebrain, areas we found that the cutoff points were similar. We therefore selected an average measure of the cutoff points for these two areas to determine a definite cutoff point (amplitude cutoff point: 0.17 for subject 1 , retinotopy; 0.19 for subject 1 , morph stimuli; 0.17 for subject 2, retinotopy, and 0.19 for subject 2, morph stimuli). Fig. 4 shows an illustration of a thresholded amplitude map for the morphing data for one subject. These amplitude maps were used to threshold the strongest phase responses that will be presented in the results section.

Note that we use the total Fourier power to divide the amplitude at the signal frequency with. It is also possible to restrict the calculation of the total Fourier power to certain frequencies by excluding the low frequencies and harmonics of the stimulus frequency, as has been done previously (Saygin and Sereno, 2008). This would increase the power in all of the maps, so it is important to take into account when comparing amplitude values across studies. For example, this corrected amplitude for the morph series in FFA would increase the average amplitude from 0.22 to 0.40 . However, there is less variation among methods in terms of statistical significance, given that the cutoff points based on the white matter and the outside-brain regions also increase when the calculation of total Fourier power is based on less frequencies.

\section{Pilot study}

In a behavioral pilot study we tested for a possible 'hysteresis' effect for the morph stimuli, namely whether the categorization shift (perceiving stimuli as either a house or a face) occurs at different times depending on how the sequence is presented (house-face or face-house). Another question was whether the orientation in which the stimuli were presented influenced the results, because these different orientations would be grouped together in the fMRI study. The structure of the pilot study matched the fMRI study closely, with only some minor alterations. The experiments were shown on a CRT monitor (resolution $1024 \times 768$, refresh rate $75 \mathrm{~Hz}$ ). The room was darkened during the experiment, and distance to the screen was approximately $50 \mathrm{~cm}$. The subjects were first made familiar with the stimuli used, by randomizing all the images and showing them in quick succession to the subjects. In the pilot study, for each

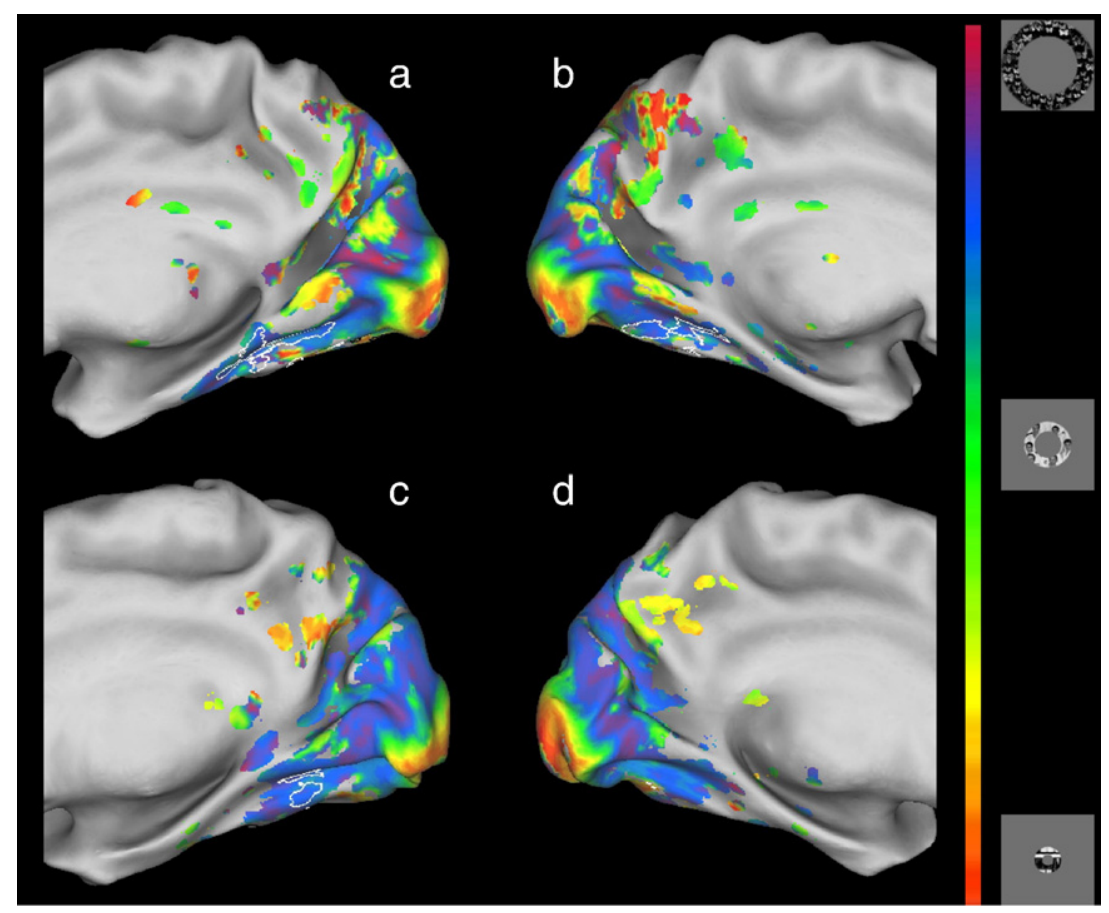

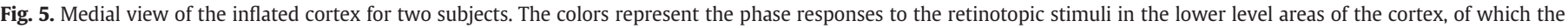

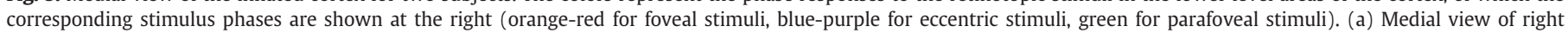
hemisphere for subject 1. (b) Medial view of left hemisphere for subject 1. (c) Medial view of right hemisphere for subject 2. (d) Medial view of left hemisphere for subject 2. 
orientation ( $45^{\circ}$ left, $45^{\circ}$ right and front view), the morph series was shown in each possible direction (from face to house or from house to face), and repeated 4 times per run. These combinations were repeated once, resulting in 12 runs in total. Presentation order of the runs was counter-balanced across subjects. The presentation parameters (stimuli per cycle, duration of stimuli and inter-stimulus interval) were exactly the same as in the fMRI experiment. At the beginning and the end of the run there was a fixation period of $5 \mathrm{~s}$. Subjects were asked to press a button each time they perceived a shift from a face to a house or from a house to a face in the morph series (excluding the sudden shifts that occur at the beginning of a new cycle). We numbered the images from 1 to 24 , with $100 \%$ house being number 1 , and $100 \%$ face being number 24 .

A repeated measures ANOVA showed that the stimulus at which the switch in perceived category occurred depended on the presentation order $(F(1,9)=9.5, p=0.013)$ : The shift from house to face occurred at a lower stimulus number (at stimulus 10 , $S E M=0.82$ ) than the shift from face to house (at stimulus 13 , $S E M=0.95)$. However, since the mean stimuli for both directions were located closely to the mean stimulus (stimulus 12), and the absolute difference in mean stimulus at which the switch occurred (two stimuli difference) was not large, we did not expect substantial effects on the fMRI study that would preclude the averaging of fMRI data across both stimulus orders. Most importantly, given the timing of the stimuli the hysteresis effect gives a deviation of the perceived turnover point from face to house (or vice versa) of only 1 to $2 \mathrm{~s}$, and this is markedly smaller than the hemodynamic lag of $5 \mathrm{~s}$ that is neutralized by doing this averaging (see section on fMRI analysis). Thus, a small hysteresis effect exists but not to an extent that it would hamper the averaging of fMRI data across both directions. There was no effect of orientation for the pilot study $\left(M_{\text {right }}=11.8, M_{\text {left }}=11.4\right.$, $\left.M_{\text {front }}=12.1 ; F(2,18)=1.98, p=0.17\right)$. This allows us to group the results of the three different orientations in one analysis. Finally, there was no interaction effect between orientation of the stimuli and the direction in which they were shown $(F(2,18)=0.81, p=0.46)$.

\section{Results}

\section{Results for the retinotopic stimuli}

In the lower visual areas we find a topographic map of eccentricity consistent with earlier results (see e.g. Engel et al., 1994; Sereno et al., 1995; Tootell et al., 1997, Wandell, 1999). Fig. 5 shows a clear gradual organization based on eccentricity visible in the medial view for both subjects and this in both hemispheres. The gradual nature of the eccentricity shifts is a basic characteristic of this topographic mapping.

In the occipitotemporal cortex, seen clearly in the ventral inflated views of the cortex, we found a link between eccentricity and the ROIs, consistent with findings from Hasson et al. (2002). Though the link is by no means absolute, there is the expected relationship between FFA and foveal activation, and PPA and eccentric activation. Fig. 6 shows this relationship clearly. In both hemispheres, and for both subjects, there is a correspondence between the face-selective areas (black outlines) and the phase responses to foveal stimuli, as well as a correspondence between the house-selective areas (in white) and the phase responses to the eccentric stimuli. Note that there is not always an exact match between the block-design data and the phase responses for eccentricity (example differences are marked with a black arrow). When comparing the eccentricity phase responses for the two subjects in FFA and PPA in the left and right hemisphere ( 4 hemispheres in total, with the mean values in FFA and PPA in each hemisphere used as a separate data point), we found a significant difference between FFA and PPA $(p<0.01, t$-test with $N=4$ ). We also calculated the PSC values for the most and least preferred stimuli based on the average response amplitude in each ROI (see Materials and methods section). In FFA we found a mean PSC

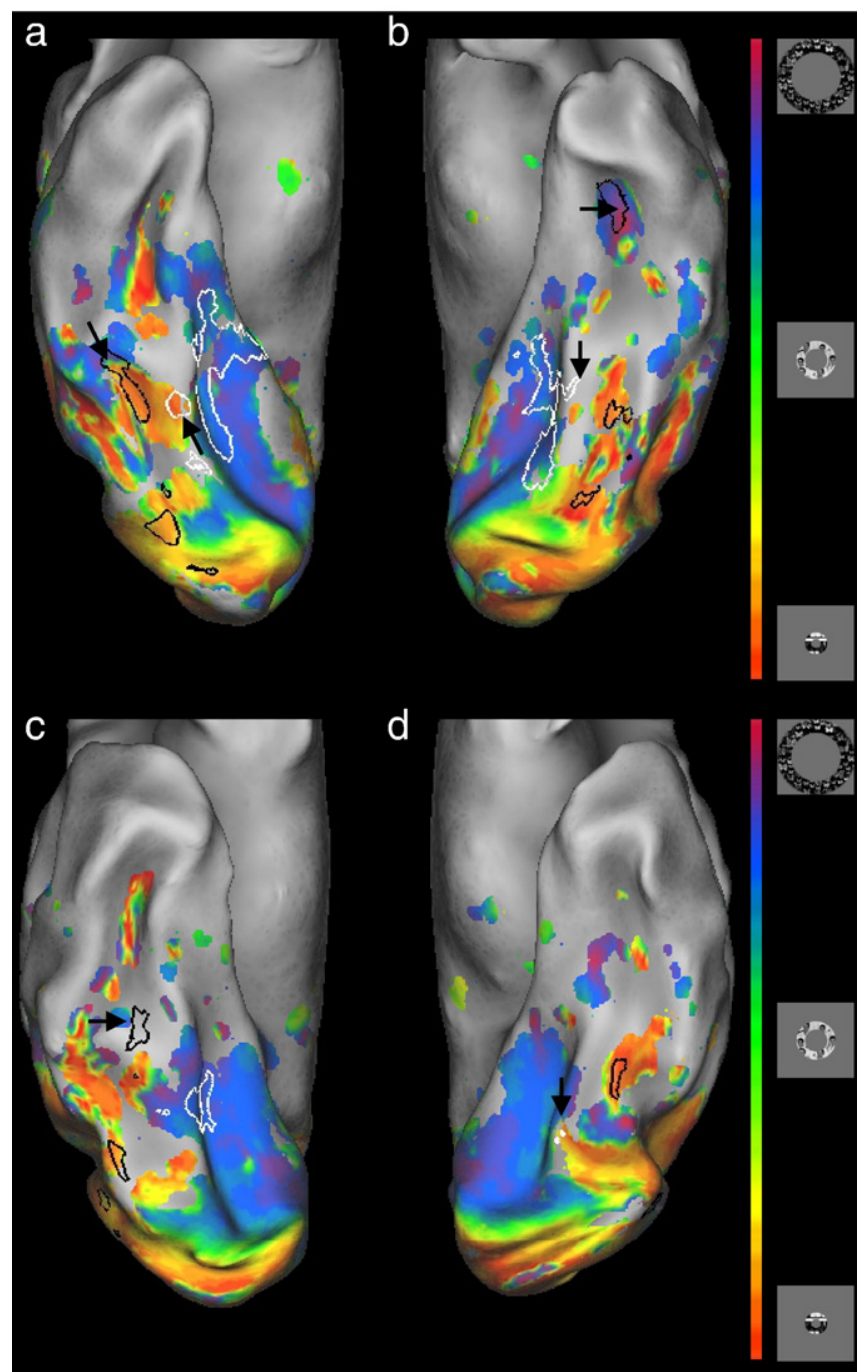

Fig. 6. Ventral view of the inflated cortex for two subjects. FFA is marked with a black line, PPA with a white line. Note that FFA and PPA are always present, though the area is sometimes very small (PPA in (d)). The colors represent the phase responses to the retinotopic stimuli, of which the corresponding stimulus phases are shown at the right (orange-red for foveal stimuli, blue-purple for eccentric stimuli and green for parafoveal stimuli). (a) Ventral view of the right hemisphere for subject 1. (b) Ventral view of the left hemisphere for subject 1. (c) Ventral view of the right hemisphere for subject 2. (d) Ventral view of the left hemisphere for subject 2. The black arrows emphasize the patches where we see a deviation from the general link between FFA/PPA and foveal/ eccentric representations, respectively.

of 1.09 across subjects for the most preferred stimulus, and a mean PSC of 0.68 across subjects for the least preferred stimulus. In PPA the values were 1.71 PSC for the most preferred and 1.20 for the least preferred stimulus.

This does not result, however, in an extended/large-scale retinotopic map stretching from FFA to PPA. There is no strong activation in the part of cortex between these regions to the intermediate stimuli, in fact no response to these stimuli is found above threshold. It is very likely that smaller retinotopic maps exist in the high-level visual areas (Wandell et al., 2007), in fact, some studies have extended retinotopic mapping to study higher-level areas (see Saygin and Sereno, 2008 for parietal cortex and Arcaro et al., 2009; Brewer et al., 2005) for retinotopy in ventral/occipital cortex), and found evidence for smaller-scale maps. The scope of this study however was limited to examining larger-scale retinotopic maps spanning FFA and PPA, and no such gradual maps comparable to the maps in the lower visual areas can be found. 
Results for the morphed house-face stimuli

Again a ventral view is presented, showing the regions of interest (Fig. 7). In this case there is a good match between the phaseencoding data and the previously created ROIs based on a blocked design. There is a clear overlap between the phase responses for the face stimuli and FFA, and the house stimuli and PPA, seen in Fig. 7, with FFA again marked in black and PPA in white. This overlap is present, even when the corresponding areas are very small (e.g. very small PPA in Fig. 7d). This overlap is significant when we compare the phase responses in all four hemispheres $(p<0.001$ for $N=4)$. This finding confirms that the newly developed technique succeeded in establishing a preference for faces and houses in the occipitotemporal cortex. Since the ROIs were defined using data from an earlier experiment involving a block design, completely unconnected to this study, with data obtained several months earlier and with very different face and house exemplars, this match can be considered particularly successful.

We also calculated PSC values for the least and most preferred stimuli in FFA and PPA. For FFA, we found mean PSC values across

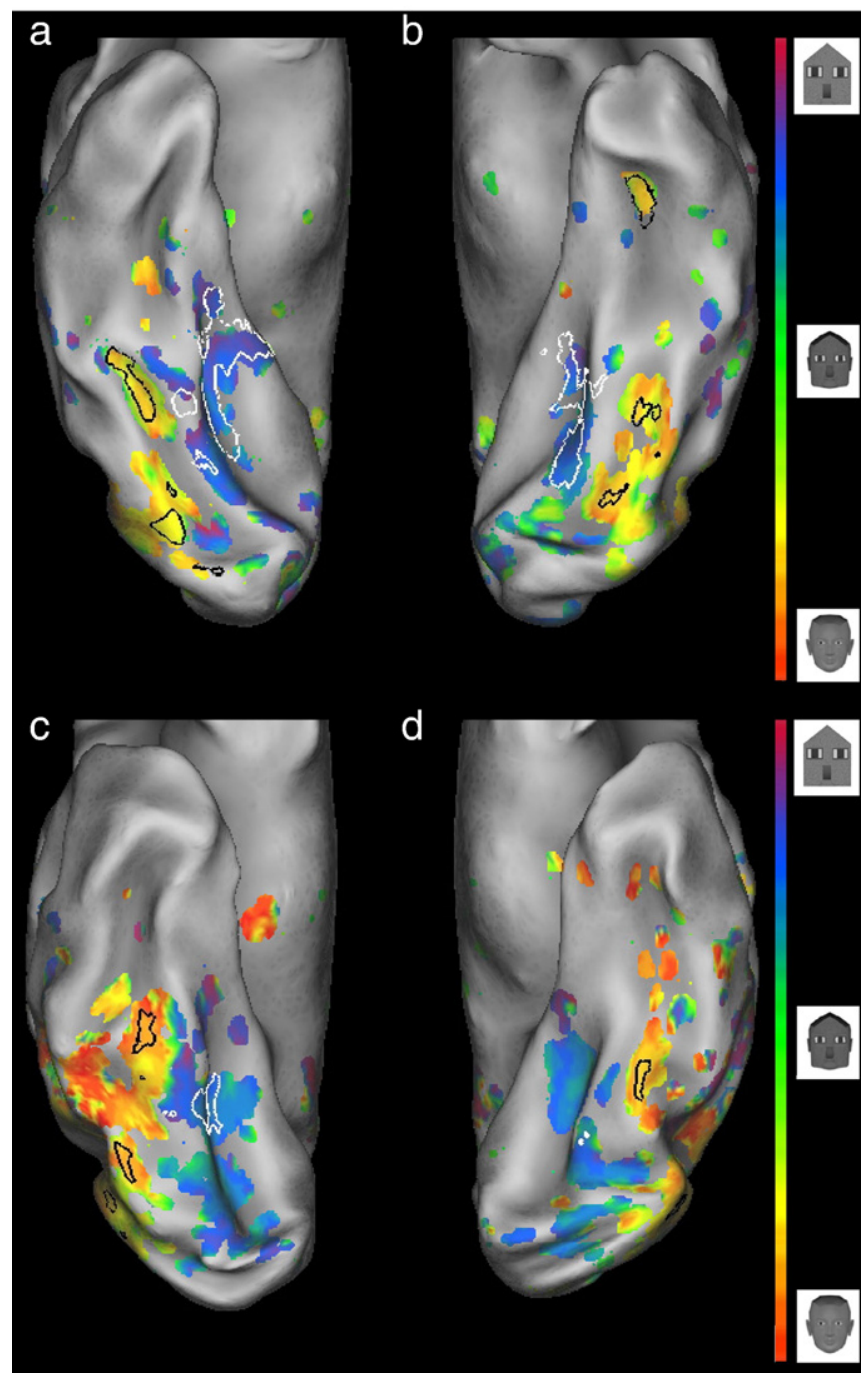

Fig. 7. Ventral view of the inflated cortex for two subjects. FFA is marked with a black line, PPA with a white line. Note that FFA and PPA are always present, though the area is sometimes very small (PPA in (d)). The colors represent the phase responses to the morphed stimuli, of which the corresponding stimulus phases are shown at the right (orange-red for faces, blue-purple for houses and green for the morphs). (a) Ventral view of the right hemisphere for subject 1. (b) Ventral view of the left hemisphere for subject 1. (c) Ventral view of the right hemisphere for subject 2. (d) Ventral view of the left hemisphere for subject 2 .

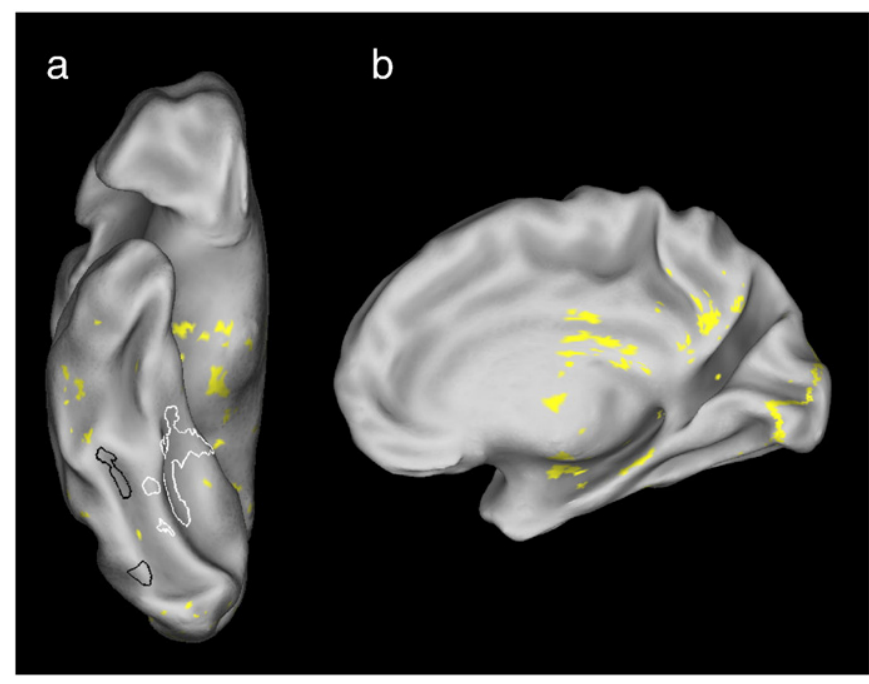

Fig. 8. Responses to the intermediate stimuli for subject 1. (a) The inflated right hemisphere in a ventral view. FFA is shown in black, PPA in white. The (unthresholded) responses to the intermediate morph stimuli are marked in yellow. (b) The inflated right hemisphere in the medial view. Here responses to the intermediate retinotopic stimuli are marked.

subjects of 1.42 for the most preferred stimulus, and 0.90 for the least preferred stimulus. In PPA, we found PSC values of 0.56 for the most preferred and 0.12 for the least preferred stimulus.

On the matter of the existence of a large-scale topographic map spanning FFA and PPA, the results show, as with the retinotopic data, a fragmented view of phase responses on the cortex. Even when taking into account the unthresholded responses we only found responses locked to the intermediate morph stimuli in the lower visual areas, meaning that there seems to be no large-scale intermediate activation in the occipitotemporal cortex. This can be seen in Fig. 8. It shows a medial and ventral view of the right hemisphere of one subject, and again FFA and PPA are marked on the cortex. It shows a lack of intermediate responses to the morph stimuli between FFA and PPA in the ventral view, and a clear band of intermediate responses to the parafoveal retinotopic stimuli in the medial view. Again, taking into account that the intermediate regions do not reach the threshold, and there is little to no activation found for the intermediate stimuli in these areas when looking at the unthresholded data, it seems that no large-scale topographic organization connects these category-selective regions.

\section{Discussion}

Our results show, as expected, a clear retinotopic map for eccentricity in the lower level visual areas. In the occipitotemporal cortex, we did successfully encode the activity in the ROIs using the traveling wave technique. We found a correspondence between FFA and phase responses to the face-like stimuli, as well as between PPA and phase responses to the house-like stimuli. In addition, we also found a similar correspondence between FFA and PPA and foveal and eccentric stimuli, respectively, confirming findings by Levy et al. (2001) and Hasson et al. (2002). However, we did not find any largescale gradual maps in occipitotemporal cortex spanning FFA, PPA, and the intermediate cortex.

When looking at the morphed object stimuli, we found a clear link between the block data and the phase-encoding data: phase responses to faces are located in and around FFA, and those to houses in and around PPA. These results are the more interesting given that the block data used to make this comparison were collected in another study and with different stimuli, thus in a completely different setting than the phase-encoding data. As with the 
retinotopic data in the ventral visual stream, we did not find a clear topographic map: There were no clear responses to the intermediate stimuli, even when looking at the unthresholded activation maps. The few intermediate responses we found in these unthresholded maps are not located between the two regions of interest.

Whereas the use of phase-encoding techniques to investigate high-level visual properties of stimuli is new, a morphed continuum between a face and a building has been included in one other recent study (Tootell et al., 2008). This study included a standard block design. It also used a different morphing approach where no corresponding features were used. As a consequence, the stimuli in the middle of the morph continuum were unidentifiable as a facehouse morph. In our case, the middle morphs are recognizable as faceand house-like, and they are ambiguous stimuli: They can be seen as either a house or a face. It is not clear which type of morphing is preferable, and morphing between complex stimuli can be done in many different ways. However, these many different types of morphing can be grouped according to the characteristics of the intermediate morphs: It can be unidentifiable, or difficult to interpret morphs, and identifiable morphs, meaning morphs that include clear features of both extremes. Taken together, this study, and that of Tootell et al. (2008) provide evidence that two very different morphing algorithms result in the same finding, namely an absence of a topographic map related to the degree of morphing.

Looking at the range of evidence we collected: There were strong differences in phase responses between FFA and PPA, even with our low number of subjects; no indication of a response between FFA and PPA for the intermediate stimuli, even when looking at belowthreshold phase responses; and lack of intermediate activation between FFA and PPA, regardless of the morphing stimuli or method used, there seems to be strong indications pointing to a more modular organization of the ventral visual cortex.

Another feature of our current design that sets it apart from previous work is the explicit comparison of the functional organization for complex face-house morphs with the organization for the more simple property of stimulus eccentricity. This comparison allowed us to investigate whether the degree of topographic organization in occipitotemporal cortex is different for category selectivity than for eccentricity. We did not find any difference, and no topographic organization was found for either property, category membership or eccentricity. The category selectivity and the eccentricity preference seem to reflect the same underlying functional organization, which is an organization in disparate functional units.

The findings showed a clear retinotopic map (or at least the fovealto-periphery aspect of it) in the primary visual cortex, confirming previous studies (see e.g. Sereno et al., 1995; Tootell et al., 1997), even though the technique used here did not include a standard checkerboard pattern. This is similar to findings by Saygin and Sereno (2008), where other stimuli besides checkerboards were used in the rotating wedges and expanding/contracting rings. It is not surprising that the finding of retinotopic maps is not limited to the typical V1sensitive stimuli, but other stimuli, more adapted to other brain regions, can be used as well.

In the occipitotemporal cortex we found supporting evidence for the findings by Levy et al. (2001) and Hasson et al. (2002) that a correspondence exists between eccentricity and the type of stimulus: Phase responses for eccentric stimuli were found in and around the PPA, and the phase responses for the foveal stimuli were found around FFA. Just as the previously reported data did not show an absolute division, we did not find one here: Though the general phase response in the ROIs corresponds to either foveal (FFA) or eccentric (PPA) activation, a close inspection of the maps suggests that there are some sub-regions where this does not hold. Most importantly, our study adds the important information that this eccentricity map is not a large-scale gradual map spanning FFA, PPA and other categoryselective regions: The intermediate cortical regions between FFA and
PPA showed little or no response in general, and even less response to the intermediate rings. Where the findings of Levy et al. (2001) found some suggestion of a larger-scale retinotopic map, we found that the organization of eccentricity in these areas is weak, and seems to be focused around the central and peripheral representations, a possibility also suggested by Malach et al. (2002).

While our results did not show large-scale topographic maps spanning FFA and PPA, this does not exclude the possibility of smallerscale maps in high-level visual cortex. For example, previous studies found evidence for retinotopy in LOC (Larsson and Heeger 2006; Wandell et al., 2007). While evidence seems to suggest that the cortical object vision pathway contains separate modules, those modules could still be made up of small retinotopic or other topographic maps, and those could be defined using proper phaseencoding techniques. For retinotopy, a very recent study has indeed provided evidence for small eccentricity and polar angle maps in the PPA (Arcaro et al., 2009). The new morphing paradigm could be used to investigate other possible object properties within certain objectselective areas, and may be of help to define additional topographic maps.

Summarizing, the use of phase-encoding techniques in this study provided us with further evidence to support the results of previous phase-encoding and block-design studies (e.g. Engel et al., 1994; Epstein et al., 1999; Kanwisher et al., 1997; Tootell et al., 2008, Sereno et al., 1995, Wandell, 1999). We found a large retinotopic map for eccentricity in the lower visual areas. While no large gradual or retinotopic maps could be established in the high-level visual cortex, we did find significant correspondence between traditional blocked designs, traditional phase-encoding, and the phase-encoding techniques we developed for this study: Activity in FFA was locked to the face-like and foveal stimuli, and activity in PPA was locked to the house-like and eccentric stimuli. Further studies into the smaller-scale organization of these areas could still reveal smaller maps, as seems to be the case in LOC (Larsson and Heeger 2006; Wandell et al., 2007). In addition, phase-encoding techniques could now also be used to further study other aspects of object perception, which were previously limited to blocked designs.

\section{Acknowledgments}

We thank M. Van Baelen, B. Vermaercke, and Ron Peeters for technical assistance with the experiment, G. d'Ydewalle and J. Wagemans for advice and support, and C. Gillebert for helpful comments on the manuscript. This work was supported by a federal research action (IUAP P6/29), and the Fund for Scientific ResearchFlanders (1.5.022.08).

\section{References}

Arcaro, M.J., McMains, S.A., Singer, B.D., Kastner, S., 2009. Retinotopic organization of human ventral visual cortex. J. Neurosci. 29, 10638-10652.

Brainard, D.H., 1997. The psychophysics toolbox. Spatial Vision 10, 443-446.

Brewer, A.A., Liu, J.J., Wade, A.R., Wandell, B.A., 2005. Visual field maps and stimulus selectivity in human ventral occipital cortex. Nat. Neurosci. 8, 1102-1109.

DeYoe, E.A., Bandettini, P., Neitz, J., Miller, D., Winans, P., 1994. Functional magnetic resonance imaging (FMRI) of the human brain. J. Neurosci. Methods 54, 171-187.

Downing, P.E. Jiang, Y.H., Shuman, M., Kanwisher, N., 2001. A cortical area selective for visual processing of the human body. Science 293, 2470-2473.

Engel, S.A., Rumelhart, D.E., Wandell, B.A., Lee, A.T., Glover, G.H., Chichilnisky, E.J., Shadlen, M.N., 1994. Fmri of human visual cortex. Nature 369, 525

Engel, S.A., Glover, G.H., Wandell, B.A., 1997. Retinotopic organization in human visual cortex and the spatial precision of functional MRI. Cereb. Cortex 7, 181-192.

Epstein, R., Kanwisher, N., 1998. A cortical representation of the local visual environment. Nature 392, 598-601.

Epstein, R., Harris, A., Stanley, D., Kanwisher, N., 1999. The parahippocampal place area: Recognition, navigation, or encoding? Neuron 23, 115-125.

Gauthier, I., Tarr, M.J., Moylan, J., Skudlarski, P., Gore, J.C., Anderson, A.W., 2000. The fusiform "face area" is part of a network that processes faces at the individual level. J. Cogn. Neurosci. 12, 495-504.

Grill-Spector, K., 2003. The neural basis of object perception. Curr. Opin. Neurobiol. 13, 159-166. 
Grill-Spector, K., Malach, R., 2004. The human visual cortex. Annu. Rev. Neurosci. 27, 649-677.

Grill-Spector, K., Kushnir, T., Hendler, T., Edelman, S., Itzchak, Y., Malach, R., 1998. A sequence of object-processing stages revealed by fMRI in the human occipital lobe. Hum. Brain Mapp. 6, 316-328.

Hasson, U., Levy, I., Behrmann, M., Hendler, T., Malach, R., 2002. Eccentricity bias as an organizing principle for human high-order object areas. Neuron 34, 479-490.

Haxby, J.V., Ishai, A., Chao, L.L., Ungerleider, L.G., Martin, A., 2000. Object-form topology in the ventral temporal lobe-response to I. Gauthier (2000). Trends Cogn. Sci. 4, 3-4.

Kanwisher, N., 2006. Neuroscience-what's in a face? Science 311, 617-618.

Kanwisher, N., McDermott, J., Chun, M.M., 1997. The fusiform face area: a module in human extrastriate cortex specialized for face perception. J. Neurosci. 17, 4302-4311.

Larsson, J., Heeger, D.J., 2006. Two retinotopic visual areas in human lateral occipital cortex. J. Neurosci. 26, 13128-13142.

Levy, I., Hasson, U., Avidan, G., Hendler, T., Malach, R., 2001. Center-periphery organization of human object areas. Nat. Neurosci. 4, 533-539.

Malach, R., Reppas, J.B., Benson, R.R., Kwong, K.K., Jiang, H., Kennedy, W.A., Ledden, P.J. Brady, T.J., Rosen, B.R., Tootell, R.B.H., 1995. Object-related activity revealed by functional magnetic-resonance-imaging in human occipital cortex. Proc. Natl. Acad. Sci. U. S. A. 92, 8135-8139.

Malach, R., Levy, I., Hasson, U., 2002. The topography of high-order human object areas. Trends Cogn. Sci. 6, 176-184.

Martin, A., Chao, L.L., 2001. Semantic memory and the brain: structure and processes. Curr. Opin. Neurobiol. 11, 194-201.

Murray, S.O., Kersten, D., Olshausen, B.A., Schrater, P., Woods, D.L., 2002. Shape perception reduces activity in human primary visual cortex. Proc. Natl. Acad. Sci. U. S. A. 99, 15164-15169.

Op de Beeck, H.P., Baker, C.I., DiCarlo, J.J., Kanwisher, N.G., 2006. Discrimination training alters object representations in human extrastriate cortex. J. Neurosci. 26 13025-13036.

Op de Beeck, H.P., Haushofer, J., Kanwisher, N.G., 2008a. Interpreting fMRI data: maps, modules and dimensions. Nat. Rev., Neurosci. 9, 123-135.

Op de Beeck, H.P., Torfs, K., Wagemans, J., 2008b. Perceived shape similarity among unfamiliar objects and the organization of the human object vision pathway. J. Neurosci. 28, 10111-10123.
Op de Beeck, H.P., Brants, M., Baeck, A., Wagemans J., in press. Distributed subordinate specificity for bodies, faces, and buildings in human ventral visual cortex. NeuroImage. doi:10.1016/j.neuroimage.2009.11.022.

Peelen, M.V., Downing, P.E., 2007. The neural basis of visual body perception. Nat. Rev., Neurosci. 8, 636-648.

Puce, A., Allison, T., Asgari, M., Gore, J.C., McCarthy, G., 1996. Differential sensitivity of human visual cortex to faces, letterstrings, and textures : a functional magnetic resonance imaging study. J. Neurosci. 16, 5205-5215.

Saygin, A.P., Sereno, M.I., 2008. Retinotopy and attention in human occipital, temporal, parietal, and frontal cortex. Cereb. Cortex 18, 2158-2168.

Schira, M.M., Tyler, C.W., Breakspear, M., Spehar, B., 2009. The foveal confluence in human visual cortex. J. Neurosci. 29, 9050-9058.

Schwarzlose, R.F., Baker, C.I., Kanwisher, N., 2005. Separate face and body selectivity on the fusiform gyrus. J. Neurosci. 25, 11055-11059.

Sereno, M.I., 1998. Brain mapping in animals and humans. Curr. Opin. Neurobiol. 8, 188-194.

Sereno, M.I., McDonald, C.T., Allman, J.M., 1994. Analysis of retinotopic maps in extrastriate cortex. Cereb. Cortex 4, 601-620.

Sereno, M.I., Dale, A.M., Reppas, J.B., Kwong, K.K., Belliveau, J.W., Brady, T.J., Rosen, B.R., Tootell, R.B.H., 1995. Borders of multiple visual areas in humans revealed by functional magnetic resonance imaging. Science 268, 889-893.

Tootell, R.B.H., Dale, A.M., Mendola, J.D., Hadjikhani, N., Brandt, S.A., 1997. Functional organization of human visual cortex revealed by fMRI. Invest. Ophthalmol. Visual Sci. 38, 2273.

Tootell, R.B., Devaney, K.J., Young, J.C., Postelnicu, G., Rajimehr, R., Ungerleider, L.G., 2008. fMRI mapping of a morphed continuum of 3D shapes within inferior temporal cortex. Proc. Natl. Acad. Sci. U. S. A. 105, 3605-3609.

Wandell, B.A., 1999. Computational neuroimaging of human visual cortex. Annu. Rev. Neurosci. 22, 145-175

Wandell, B.A., Wade, A.R., 2003. Functional imaging of the visual pathways. Neurol. Clin. 21, 417-443.

Wandell, B.A., Dumoulin, S.O., Brewer, A.A., 2007. Visual field maps in human cortex. Neuron 56, 366-383.

Warnking, J., Dojat, M., Guérin-Dugué, A., Delon-Martin, C., Olympieff, S., Richard, N., Chéhikian, A., Segebarth, C., 2002. fMRI retinotopic mapping step by step. NeuroImage 17, 1665. 